COMPUTATIONAL METHODS IN APPLIED MATHEMATICS, Vol. 4 (2004), No. 1, pp. 3-22

(C) 2004 Editorial Board of the Journal "Computational Methods in Applied Mathematics" Joint Co. Ltd.

\title{
OVERLAPPED BEM-FEM AND SOME SCHWARZ ITERATIONS $^{1}$
}

\author{
RICARDO CELORRIO \\ Dep. Matemática Aplicada, Universidad de Zaragoza, \\ EUITIZ, 50018 Zaragoza, Spain \\ VÍCTOR DOMÍNGUEZ \\ Dep. Matemática e Informática, Universidad Pública de Navarra \\ Campus de Arrosadía s/n, 31.006 Pamplona, Spain \\ FRANCISCO-JAVIER SAYAS \\ Dep. Matemática Aplicada, Universidad de Zaragoza \\ C.P.S., 50018 Zaragoza, Spain
}

\begin{abstract}
In this work we consider the numerical solution of the Laplace's equation in a domain with holes by means of the overlapping of finite and boundary elements. The essence of the method is the consideration of the finite element solution of the Laplace's equation in the domain without holes and the exterior single-layer solution on the unbounded domain around these holes. This solution can be viewed as a limit of a discretized interior-exterior Schwarz-type iteration. A convergence analysis of both the iteration and the discrete solution is carried out, taking full generality in the BEM scheme. Some numerical experiments are also given.
\end{abstract}

2000 Mathematics Subject Classification: 65N30, 65N38, 65F10.

Keywords: finite elements, boundary elements, Schwarz method.

\section{Introduction}

The present work is concerned with the numerical solution of a model problem, namely Laplace's equation with Dirichlet boundary conditions, on a domain with holes (henceforth referred to as obstacles), in two or three dimensions. The basic idea is to take out the interior obstacles and make a triangulation of the simpler remaining domain using finite elements on it. At the same time we will consider problems exterior to the set of boundaries of the obstacles and use boundary elements based on an indirect single layer potential formulation for this kind of problem. Then we iterate between both problems, interchanging traces, i.e., the finite element solutions generate traces on the boundaries of the obstacles and the boundary element solution is plugged into the single layer potential to obtain values on the outer boundary. The iteration is then carried out till the process becomes stationary.

\footnotetext{
${ }^{1}$ Research partially supported by MCYT Project BFM2001-2521 and MAT2002-04153
} 
The proposed method is a discrete version of the interior-exterior Schwarz iteration studied in [3]. As a scheme that iterates between the intersection of two domains, this method can be understood in the frame of classical Schwarz-Neumann iterations (see [11]). In fact, written in the adequate variables, the iteration is just a Neumann iteration for an operator equation. The kind of ideas of this method is heavily related to multiple scattering techniques and somewhat resembles Chimera type methods [2]. Just for this model situation, the method provides a simple discretization of a problem in a domain with a complicated geometry by taking out holes (in fact we do not have to eliminate all obstacles, just the part of them we choose).

For the discretization of the interior problem we consider $\mathbb{P}_{k}$ finite elements on a simplicial triangulation. The reason for this restriction is more on the analysis side of the problem than on practical issues, basically since we want to use Scott-Zhang's operator [19]. All what is used of these finite elements is stated in Appendix, so all results are valid as long as we can prove similar bounds for other finite element spaces. For the boundary element part, we consider any convergent Galerkin method, be it based on finite elements or not. Part of the analysis requires a somewhat stronger approximation property of the discrete space. This property is however satisfied by all relevant boundary element spaces.

In fact, the work develops two different approaches to the problem. First we study the iteration and prove that in some situations it has a limit. On the other hand, we can ignore the iterative process and study its possible fixed point even when this is not reached as the limit. This second approach allows to think of not only better iterations (based on GMRES, for instance), but also of more general elliptic problems.

It is the authors' belief that this paper deals with the unexplored possibility of taking out obstacles from complicated domains by using boundary layer potentials and as such opens up a chance of combining finite and boundary elements in a nonstandard and satisfying fashion. For instance, as we show in the numerical examples, this allows to use a fast Poisson solver in a rectangle by previously removing all interior obstacles with a single layer potential. The idea of analysing the whole method by taking traces as unknowns (note that the unknown on the interior boundary is a density) is another novelty of our approach and shows how to profit from boundary element and finite element techniques to deal with a nonstandard situation.

Although the method is based on the superposition principle and its applicability is thus limited to linear problems (these include iterations for some nonlinear problems), the advantage of having different grids for the whole domain and the obstacle is certainly promising for future applications in the realm of inverse problems, where interior geometries are unknown. In this situation, the boundary element grid would move without the necessity to create a new finite element grid, obliged to adapt to a possibly complicated geometry. This is the aim of a future work.

Notice also that in recent years the overlapping and non-overlapping domain decomposition methods, especially the BEM-FEM Schwarz iterations and the Dirichlet-to-Neumann method for exterior problems (unlike in our situation) have been developed in [22-25].

The paper is organized as follows. In Section 2, we formulate the model problem, describe the Schwarz BEM-FEM iteration, and write the discrete equations satisfied by its possible limit. In Section 3, we write the problem and the iteration as an equivalent set of operator equations, traces of the uncoupled problems becoming now the unknowns. This makes it possible to study the problem in relation to the continuous interior-exterior Schwarz iteration of [3]. Assuming only the convergence of the Galerkin BEM and the refinement of the interior 
triangulation, we show in Section 4 that the discrete iteration converges in all the cases where the continuous iteration converges.

In Section 5, we prove uniform boundedness of the inverse of the discrete operator obtained in Section 3. This gives an alternative proof of the same fact for the three-dimensional case (where everything works before discretization) and in the good two-dimensional cases. It also extends the result to some two-dimensional cases. The proof does not use the fact that the spectral radius of a certain operator obtained in Section 3 is less that one, but relies on Fredholm properties of this operator. This opens some expectations on the possibility of extending the results to more general situations.

In Section 6, we give an asymptotic analysis of the error as the discretization (both finite and boundary element) becomes finer. We comment on possible choices for the BEM spaces. Section 7 is devoted to the illustration of some of the results by a simple two-dimensional example, using a nonconforming Dirac delta scheme (a quadrature method) for the boundary integral part.

Appendix presents some results concerning mainly the convergence and stability of the finite element method in Sobolev spaces of fractional order.

Notational foreword. We will make extensive use of the Sobolev spaces $H^{s}(\mathcal{O})$, where $\mathcal{O}$ is an open set in $\mathbb{R}^{d}$ and $\mathbb{R} \ni s \geqslant 0$. Their norms will be denoted by $\|\cdot\|_{s, \mathcal{O}}$. For Lipschitz curves or surfaces $\Theta$, we also will consider the Sobolev spaces of real order $H^{s}(\Theta)$ with $s \in[-1,1]$, with similar notations for their norms. The definitions and properties of these spaces in the form needed here can be found in $[7,13]$.

$C$ (with possible sub- and superscripts) will denote a positive constant independent of the discretization parameter $h$ and of all quantities it is multiplied by, possibly different in each occurrence.

\section{Description of the method}

Let $Q \subset \mathbb{R}^{d}(d=2,3)$ be a domain with a polygonal (polyhedral) boundary $\Sigma$ strictly containing a possibly nonconnected open set with a Lipschitz boundary $\Gamma$. The corresponding anular domain, exterior to $\Gamma$ and interior to $\Sigma$ will be denoted by $\Omega$.
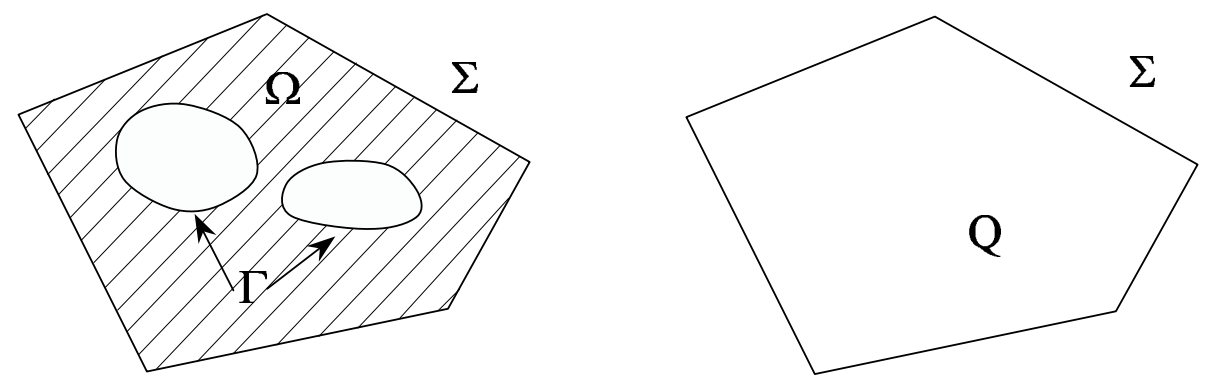

Figure 1. The domain with and without holes

The method we propose here is a Schwarz-type iteration for the boundary value problem: find $w \in H^{1}(\Omega)$ such that

$$
\mid \begin{gathered}
\Delta w=0, \quad \text { in } \Omega, \\
\gamma_{\Sigma} w=g_{\Sigma}, \quad \gamma_{\Gamma} w=g_{\Gamma}
\end{gathered}
$$


for given $g_{\Sigma} \in H^{1 / 2}(\Sigma), g_{\Gamma} \in H^{1 / 2}(\Gamma), \gamma_{\Sigma}$ and $\gamma_{\Gamma}$ being the corresponding trace operators.

In connection with the boundary $\Gamma$ we consider the single-layer potential

$$
\mathcal{S}_{\Gamma} \psi:=\int_{\Gamma} \Phi(\cdot, y) \psi(y) \mathrm{d} \sigma(y): \mathbb{R}^{d} \rightarrow \mathbb{R}
$$

for an arbitrary density $\psi \in H^{-1 / 2}(\Gamma)$, being $\Phi(x, y):=1 /|x-y|$ in three dimensions and $\Phi(x, y):=-\log |r(x-y)|$ in two dimensions. The choice of the parameter $r>0$ has some effects on the properties of the layer potentials (see Proposition 2.1 below).

We also consider the single-layer operator $V_{\Gamma}: H^{-1 / 2}(\Gamma) \rightarrow H^{1 / 2}(\Gamma)$

$$
V_{\Gamma} \psi:=\gamma_{\Gamma} \mathcal{S}_{\Gamma} \psi=\int_{\Gamma} \Phi(\cdot, y) \psi(y) \mathrm{d} \sigma(y): \Gamma \rightarrow \mathbb{R} .
$$

For the properties of this operator and the restrictions-extensions to other Sobolev spaces of real order, see $[5,13]$. In two dimensions, the concept of the logarithmic capacity of a curve or set of curves is relevant in the properties of the inversibility of $V_{\Gamma}$. For its definition, see $[13,20]$. We gather in the following proposition some well-known properties of $V_{\Gamma}$ (see [13, Ch. 8], for instance).

Proposition 2.1. In the three dimensional case, $V_{\Gamma}$ is elliptic and therefore inversible in $H^{-1 / 2}(\Gamma)$. In two dimensions, $V_{\Gamma}$ is inversible if and only if the logarithmic capacity of $\Gamma$ differs from $1 / r$ and elliptic if and only if the capacity is less than $1 / r$.

The method we propose needs two families of discrete spaces, one defined in $Q$ (a domain without holes) and the other on the boundary $\Gamma$. For simplicity, we will consider a single discretization parameter $h:=\left(h_{Q}, h_{\Gamma}\right) \rightarrow 0$, grouping the refinement possibilities of both families. The parameter $h_{\Gamma}$ does not necessarily have a geometrical meaning. In the last part of this work (Section 6) we will separate the effects of discretizations in $Q$ and $\Gamma$.

Let $\mathcal{T}_{h}$ be a regular family of simplicial triangulations in the usual sense (see $[1,6]$ ) and let $P_{h}^{Q} \subset H^{1}(Q)$ be a polynomial finite element space associated with this triangulation

$$
P_{h}^{Q}:=\left\{u_{h} \in \mathcal{C}(\bar{Q})\left|u_{h}\right|_{K} \in \mathbb{P}_{k}, \quad \forall K \in \mathcal{T}_{h}\right\}
$$

We will not make apparent the degree of the polynomials $(k \geqslant 1)$ in the definitions, since it does not appear in the analysis until the moment when we consider convergence orders. The finite element space restricted to the boundary

$$
X_{h}^{\Sigma}:=\gamma_{\Sigma} P_{h}^{Q}
$$

will be of relevance in the sequel. In connection with these spaces, we have the finite element procedure FEM : $X_{h}^{\Sigma} \rightarrow P_{h}^{Q}$

$$
\operatorname{FEM}\left(g_{h}^{\Sigma}\right)=u_{h}, \quad \mid \begin{array}{cl}
u_{h} \in P_{h}^{Q}, & \gamma_{\Sigma} u_{h}=g_{h}^{\Sigma}, \\
\int_{Q} \nabla u_{h} \cdot \nabla v_{h}=0, & \forall v_{h} \in P_{h}^{Q} \cap H_{0}^{1}(Q) .
\end{array}
$$

For the interior boundary we consider the family of subspaces $S_{h}^{\Gamma} \subset H^{-1 / 2}(\Gamma)$ under conditions to be fixed later. In general, this space can be composed of finite elements, trigonometric 
polynomials (in 2 dimensions) or spherical harmonics (in 3 dimensions), etc. We then define the Galerkin boundary element procedure: BEM : $H^{1 / 2}(\Gamma) \rightarrow S_{h}^{\Gamma}$

$$
\operatorname{BEM}\left(g_{\Gamma}\right)=\psi_{h}, \quad \mid \begin{aligned}
& \psi_{h} \in S_{h}^{\Gamma}, \\
& \left\langle V_{\Gamma} \psi_{h}, \xi_{h}\right\rangle=\left\langle g_{\Gamma}, \xi_{h}\right\rangle, \quad \forall \xi_{h} \in S_{h}^{\Gamma},
\end{aligned}
$$

being $\langle\cdot, \cdot\rangle$ the $H^{1 / 2}(\Gamma) \times H^{-1 / 2}(\Gamma)$ duality product.

Finally, to define the iterative process, we introduce the operator $V_{\Sigma \Gamma}: H^{-1 / 2}(\Gamma) \rightarrow H^{1 / 2}(\Sigma)$

$$
V_{\Sigma \Gamma} \psi:=\gamma_{\Sigma} \mathcal{S}_{\Gamma} \psi=\int_{\Gamma} \Phi(\cdot, y) \psi(y) \mathrm{d} \sigma(y): \Sigma \rightarrow \mathbb{R}
$$

and the nodal Lagrange interpolation operator $I_{h}^{\Sigma}: \mathcal{C}(\Sigma) \rightarrow X_{h}^{\Sigma}$, which is well defined for the finite elements considered above.

The iteration proceeds as follows: first, we choose

$$
X_{h}^{\Sigma} \ni g_{0, h}^{\Sigma} \approx g_{\Sigma}
$$

(how this function is chosen is not relevant for convergence of the iteration, but it is relevant for convergence as $h \rightarrow 0$ ); then we do

$$
\begin{aligned}
u_{0, h}:= & \operatorname{FEM}\left(g_{0, h}^{\Sigma}\right) \\
\psi_{0, h}:= & \operatorname{BEM}\left(g_{\Gamma}\right) \\
\text { for } n \geqslant 0 & \\
& u_{n+1, h}:=\operatorname{FEM}\left(g_{0, h}^{\Sigma}-I_{h}^{\Sigma} V_{\Sigma \Gamma} \psi_{n, h}\right)=u_{0, h}-\operatorname{FEM}\left(I_{h}^{\Sigma} V_{\Sigma \Gamma} \psi_{n, h}\right) \\
& \psi_{n+1, h}:=\operatorname{BEM}\left(g_{\Gamma}-\gamma_{\Gamma} u_{n, h}\right)=\psi_{0, h}-\operatorname{BEM}\left(\gamma_{\Gamma} u_{n, h}\right) .
\end{aligned}
$$

The approximation to the exact solution is given by the expressions

$$
w_{n, h}:=u_{n, h}+\mathcal{S}_{\Gamma} \psi_{n, h}: \Omega \rightarrow \mathbb{R} .
$$

Assuming the existence of a limit as $n \rightarrow \infty$, say $u_{h}=\lim u_{n, h}$ and $\psi_{h}=\lim \psi_{n, h}$, it is easy to show that these functions satisfy the discrete equations

$$
\left[\begin{array}{cc}
I & \mathrm{FEM} I_{h}^{\Sigma} V_{\Sigma \Gamma} \\
\operatorname{BEM} \gamma_{\Gamma} & I
\end{array}\right]\left[\begin{array}{c}
u_{h} \\
\psi_{h}
\end{array}\right]=\left[\begin{array}{c}
u_{0, h} \\
\psi_{0, h}
\end{array}\right],
$$

or equivalently

$$
\mid \begin{aligned}
u_{h} & \in P_{h}^{Q}, & & \psi_{h} \in S_{h}^{\Gamma}, \\
\left.u_{h}\right|_{\Sigma}+I_{h}^{\Sigma}\left(\left.\mathcal{S}_{\Gamma} \psi_{h}\right|_{\Sigma}\right) & =g_{h, 0}^{\Sigma}, & & \\
\int_{Q} \nabla u_{h} \cdot \nabla v_{h} & =0, & & \forall v_{h} \in P_{h}^{Q} \cap H_{0}^{1}(Q), \\
\int_{\Gamma}\left(u_{h}+V_{\Gamma} \psi_{h}\right) \xi_{h} & =\int_{\Gamma} g_{\Gamma} \xi_{h}, & & \forall \xi_{h} \in S_{h}^{\Gamma} .
\end{aligned}
$$

Iteration (4) is a block Jacobi method for (5). Note that because of its particular form, the Jacobi method is equivalent to this block partitioned method. A Gauss-Seidel type iteration is also possible, although it loses the parallel structure of the Jacobi method. 
Moreover, we can think of equations (5) as a discretization of the model problem (1) by defining finally the approximation $w_{h}=u_{h}+\mathcal{S}_{\Gamma} \psi_{h}$. We thus can solve these discrete equations by other iterative schemes requiring only multiplication by the matrix (i.e., the solution of a finite element and a boundary element problem), such as GMRES (see $[8,14])$.

\section{An equivalent formulation}

The above iterative method can be written in an equivalent way, which emphasizes its proximity to the interior-exterior Schwarz algorithm developed in [3]. This reformulation will be used to give the convergence analysis both as $n \rightarrow \infty$ and as $h \rightarrow 0$.

Let $R: H^{1 / 2}(\Sigma) \rightarrow H^{1}(Q)$ be the harmonic lifting operator

$$
\mid \begin{gathered}
R g_{\Sigma} \in H^{1}(Q), \quad \gamma_{\Sigma} R g_{\Sigma}=g_{\Sigma}, \\
\int_{Q} \nabla R g_{\Sigma} \cdot \nabla v=0, \quad \forall v \in H_{0}^{1}(Q) .
\end{gathered}
$$

Some relevant properties of $R$ are reviewed in Appendix. We then consider the operators $K_{\Gamma \Sigma}:=\gamma_{\Gamma} R: H^{1 / 2}(\Sigma) \rightarrow H^{1 / 2}(\Gamma)$ and $K_{\Sigma \Gamma}:=V_{\Sigma \Gamma} V_{\Gamma}^{-1}: H^{1 / 2}(\Gamma) \rightarrow H^{1 / 2}(\Sigma)$ and two matrices of the operators $\mathcal{A}, \mathcal{K}: H^{1 / 2}(\Sigma) \times H^{1 / 2}(\Gamma) \rightarrow H^{1 / 2}(\Sigma) \times H^{1 / 2}(\Gamma)$

$$
\mathcal{K}:=\left[\begin{array}{cc}
0 & K_{\Sigma \Gamma} \\
K_{\Gamma \Sigma} & 0
\end{array}\right], \quad \mathcal{A}:=\mathcal{I}+\mathcal{K}=\left[\begin{array}{cc}
I & K_{\Sigma \Gamma} \\
K_{\Gamma \Sigma} & I
\end{array}\right]
$$

It is easy to prove that $\mathcal{K}$ is compact.

Proposition 3.1 [3]. If $V_{\Gamma}$ is inversible, $\mathcal{A}$ is inversible.

Proposition 3.2 [3]. The spectral radius of the compact operator $\rho(\mathcal{K})=\left(\rho\left(K_{\Sigma \Gamma} K_{\Gamma \Sigma}\right)\right)^{1 / 2}$ is less than one: (a) in three dimensions; (b) in two dimensions if the logarithmic capacity of $\Sigma$ is less than $1 / r$.

Note that the logarithmic capacity of the complete domain is relevant in this result (however, the hypothesis can be somewhat weakened [3] Proposition 3.3). The lack of good properties for the two-dimensional case be can easily mended by taking a smaller value of $r$ or changing the units in the space to make the domain smaller.

In addition, if we consider the uniquely solvable system

$$
\left[\begin{array}{cc}
I & K_{\Sigma \Gamma} \\
K_{\Gamma \Sigma} & I
\end{array}\right]\left[\begin{array}{l}
f_{\Sigma} \\
f_{\Gamma}
\end{array}\right]=\left[\begin{array}{l}
g_{\Sigma} \\
g_{\Gamma}
\end{array}\right]
$$

then the solution to (1) can be decomposed as

$$
w=R f_{\Sigma}+\mathcal{S}_{\Gamma} V_{\Gamma}^{-1} f_{\Gamma}
$$

i.e., the equations provide a unique decomposition of $w$ as the sum of the single-layer potential plus the solution in the whole set $Q$. This idea is quite common in the field of time-harmonic waves, where $R f_{\Sigma}$ plays the role of an incident wave and the other term is the scattering produced by $\Gamma$. We will see that the iterative method can be understood as a discretization of the Jacobi method for (7) (which coincides with the Neumann series iteration), which was studied in [3]. 
New discrete spaces and operators. In order to write the iteration and its possible limit more appropriately, we introduce some new discrete elements:

(a) Let $X_{h}^{\Gamma}:=V_{\Gamma} S_{h}^{\Gamma}$. This space, isomorphic to $S_{h}^{\Gamma}$, appears just for the sake of analysis, but does not have to be constructed or used in practice.

(b) Let $R_{h}: X_{h}^{\Sigma} \rightarrow P_{h}^{Q}$ be the discrete harmonic lifting (up to now denoted by FEM). Some properties of $R_{h}$ are given in the appendix.

(c) Let $L_{h}: H^{1 / 2}(\Gamma) \rightarrow X_{h}^{\Gamma}$ be given by $L_{h}:=V_{\Gamma}$ BEM, i.e.,

$$
\mid \begin{aligned}
& L_{h} g_{\Gamma} \in X_{h}^{\Gamma}, \\
& \left\langle L_{h} g_{\Gamma}, \xi_{h}\right\rangle=\left\langle g_{\Gamma}, \xi_{h}\right\rangle, \quad \forall \xi_{h} \in S_{h}^{\Gamma} .
\end{aligned}
$$

(d) Finally, consider any projection $N_{h}: H^{1 / 2}(\Sigma) \rightarrow X_{h}^{\Sigma}$ that is uniformly bounded as an operator in $H^{1 / 2}(\Sigma)$. At this stage, the $H^{1 / 2}(\Sigma)$-orthogonal projection onto $X_{h}^{\Sigma}$ is a suitable choice for $N_{h}$. The operator itself is immaterial for what follows.

These operators lead to the consideration of the approximations of $K_{\Sigma \Gamma}$ and $K_{\Gamma \Sigma}$

$$
\begin{array}{lll}
K_{\Sigma \Gamma}^{h}:=I_{h}^{\Sigma} K_{\Sigma \Gamma} & : & H^{1 / 2}(\Gamma) \rightarrow X_{h}^{\Sigma} \subset H^{1 / 2}(\Sigma), \\
K_{\Gamma \Sigma}^{h}:=L_{h} \gamma_{\Gamma} R_{h} N_{h} & : & H^{1 / 2}(\Sigma) \rightarrow X_{h}^{\Gamma} \subset H^{1 / 2}(\Gamma),
\end{array}
$$

the corresponding matrix of operators

$$
\mathcal{K}_{h}:=\left[\begin{array}{cc}
0 & K_{\Sigma \Gamma}^{h} \\
K_{\Gamma \Sigma}^{h} & 0
\end{array}\right]
$$

and $\mathcal{A}_{h}:=\mathcal{I}+\mathcal{K}_{h}: H^{1 / 2}(\Sigma) \times H^{1 / 2}(\Gamma) \rightarrow H^{1 / 2}(\Sigma) \times H^{1 / 2}(\Gamma)$.

Proposition 3.3. Consider iteration (4) and let

$$
g_{n, h}^{\Sigma}:=\gamma_{\Sigma} u_{n, h} \in X_{h}^{\Sigma} \quad g_{n, h}^{\Gamma}:=V_{\Gamma} \psi_{n, h}^{\Gamma} \in X_{h}^{\Gamma} .
$$

Then

$$
\left[\begin{array}{l}
g_{n+1, h}^{\Sigma} \\
g_{n+1, h}^{\Gamma}
\end{array}\right]=\left[\begin{array}{c}
g_{0, h}^{\Sigma} \\
g_{0, h}^{\Gamma}
\end{array}\right]-\left[\begin{array}{cc}
0 & K_{\Sigma \Gamma}^{h} \\
K_{\Gamma \Sigma}^{h} & 0
\end{array}\right]\left[\begin{array}{l}
g_{n, h}^{\Sigma} \\
g_{n, h}^{\Gamma}
\end{array}\right] .
$$

Proof. A very simple manipulation of the expressions in the iteration (4), noting that $u_{n, h}=\operatorname{FEM}\left(g_{n, h}^{\Sigma}\right)$, shows that this one is equivalent to:

$$
\begin{aligned}
& g_{0, h}^{\Sigma} \approx \\
& g_{0, h}^{\Gamma}:=V_{\Gamma} \operatorname{BEM}\left(g_{\Gamma}\right) \\
& \text { for } n \geqslant 0 \\
& \qquad \begin{array}{l}
g_{n+1, h}^{\Sigma}:=g_{0, h}^{\Sigma}-I_{h}^{\Sigma} K_{\Sigma \Gamma} g_{n, h}^{\Gamma} \\
\qquad g_{n+1, h}^{\Gamma}:=g_{0, h}^{\Gamma}-V_{\Gamma} \operatorname{BEM}\left(\gamma_{\Gamma} \operatorname{FEM}\left(g_{n, h}^{\Sigma}\right)\right) .
\end{array}
\end{aligned}
$$

The result is then a straightforward consequence of the notations and of the fact that both $L_{h}$ and $N_{h}$ are projections onto the respective discrete spaces $X_{h}^{\Gamma}$ and $X_{h}^{\Sigma}$. 
This result shows that this is the Jacobi method for the equations (compare to (7))

$$
\left[\begin{array}{cc}
I & K_{\Sigma \Gamma}^{h} \\
K_{\Gamma \Sigma}^{h} & I
\end{array}\right]\left[\begin{array}{l}
f_{h}^{\Sigma} \\
f_{h}^{\Gamma}
\end{array}\right]=\left[\begin{array}{c}
g_{0, h}^{\Sigma} \\
g_{0, h}^{\Gamma}
\end{array}\right] .
$$

Note that the operator $\mathcal{A}_{h}$ is defined in the whole continuous space. However, when the right-hand side belongs to the discrete space (as in (8)), both the solution and all the iterations are in the discrete space. Therefore, the whole of the iterative process happens in a finite-dimensional setting.

\section{Convergence of the iterative process}

Hypothesis 1. As $h \rightarrow 0$, we assume that

$$
\inf _{\psi_{h} \in S_{h}^{\Gamma}}\left\|\psi-\psi_{h}\right\|_{-1 / 2, \Gamma} \rightarrow 0, \quad \forall \psi \in H^{-1 / 2}(\Gamma) .
$$

Since $V_{\Gamma}$ is strongly elliptic, this hypothesis is equivalent to the convergence of the boundary element discretization. When $h \rightarrow 0$, we are implicitly assuming that the triangulation becomes finer. Therefore

$$
\inf _{u_{h} \in P_{h}^{Q}}\left\|u-u_{h}\right\|_{1, Q} \rightarrow 0, \quad \forall u \in H^{1}(Q) .
$$

As a general notation, we will write for a family of operators $\Xi_{h}$ converging pointwise to $\Xi$ (i.e., $\Xi_{h} g \rightarrow \Xi g$ for all $g$ ),

$$
\Xi_{h} \stackrel{\mathrm{pt}}{\rightarrow} \Xi
$$

The arrow notation $\Xi_{h} \rightarrow \Xi$ denotes norm convergence.

Proposition 4.1. If Hypothesis 1 holds, for the families $L_{h}: H^{1 / 2}(\Gamma) \rightarrow H^{1 / 2}(\Gamma)$ and $N_{h}: H^{1 / 2}(\Sigma) \rightarrow H^{1 / 2}(\Sigma)$ we have the convergences:

$$
L_{h} \stackrel{\mathrm{pt}}{\rightarrow} I, \quad N_{h} \stackrel{\mathrm{pt}}{\rightarrow} I .
$$

Proof. The first one is an immediate consequence of the approximation property (9). Note also that (10) implies

$$
\inf _{g_{h} \in X_{h}^{\Sigma}}\left\|g-g_{h}\right\|_{1 / 2, \Sigma} \rightarrow 0, \quad \forall g \in H^{1 / 2}(\Sigma),
$$

which is equivalent to the second property.

Proposition 4.2. As a class of operators from $H^{1 / 2}(\Sigma) \rightarrow H^{1}(Q)$, we have

$$
\left(R_{h}-R\right) N_{h} \stackrel{\mathrm{pt}}{\rightarrow} 0 .
$$

Proof. Denoting $M_{h}: H^{1}(\Sigma) \rightarrow X_{h}^{\Sigma}$ to the orthogonal projection onto $X_{h}^{\Sigma}$

$$
\begin{aligned}
\left\|\left(R-R_{h}\right) N_{h} g\right\|_{1, Q} & \leqslant\left\|\left(R-R_{h}\right) N_{h}\left(I-M_{h}\right) g\right\|_{1, Q}+\left\|\left(R-R_{h}\right) N_{h} M_{h} g\right\|_{1, Q} \\
& \leqslant C\left\|g-M_{h} g\right\|_{1 / 2, \Sigma}+\left\|\left(R-R_{h}\right) M_{h} g\right\|_{1, Q} \\
& \leqslant C^{\prime}\left(\left\|g-M_{h} g\right\|_{1, \Sigma}+h^{1 / 2}\|g\|_{1, \Sigma}\right)
\end{aligned}
$$


for all $g \in H^{1}(\Sigma)$ (see Proposition 8.4). The first addendum converges to zero, since we are dealing with finite elements on the boundary with a refining set of grids. The density of $H^{1}(\Sigma)$ in $H^{1 / 2}(\Sigma)$ proves the result.

Theorem 4.1. If Hypothesis 1 holds, as $h \rightarrow 0$, we have the following convergences:

(a) $K_{\Sigma \Gamma}^{h} \rightarrow K_{\Sigma \Gamma}$

(b) $K_{\Gamma \Sigma}^{h} \stackrel{\mathrm{pt}}{\rightarrow} K_{\Gamma \Sigma}$

(c) $\mathcal{K}_{h} \stackrel{\mathrm{pt}}{\rightarrow} \mathcal{K}$,

(d) $K_{\Gamma \Sigma}^{h} K_{\Sigma \Gamma}^{h} \rightarrow K_{\Gamma \Sigma} K_{\Sigma \Gamma}$.

Proof. We first remark that $K_{\Sigma \Gamma}-K_{\Sigma \Gamma}^{h}=\left(I-I_{h}^{\Sigma}\right) K_{\Sigma \Gamma}=\left(I-I_{h}^{\Sigma}\right) \gamma_{\Sigma} \mathcal{S}_{\Gamma} V_{\Gamma}^{-1}$. Then (a) is a simple consequence of Proposition 8.8.

To prove (b), we first decompose

$$
K_{\Gamma \Sigma}-K_{\Gamma \Sigma}^{h}=K_{\Gamma \Sigma}\left(I-N_{h}\right)+\left(I-L_{h}\right) K_{\Gamma \Sigma} N_{h}+L_{h} \gamma_{\Gamma}\left(R-R_{h}\right) N_{h} .
$$

By Proposition 4.1, we have that $K_{\Gamma \Sigma}\left(I-N_{h}\right) \stackrel{\text { pt }}{\rightarrow} 0$. The compactness of $K_{\Gamma \Sigma}$ and Proposition 4.1 prove that $\left(I-L_{h}\right) K_{\Gamma \Sigma} N_{h} \rightarrow 0$ (see [12] Theorem 10.7). For the pointwise convergence of the third term in (11) we simply apply Proposition 4.2 and the uniform boundedness of $L_{h}$.

Convergence (c) is an obvious consequence of (a) and (b). Finally, to prove (d), we write

$$
K_{\Gamma \Sigma} K_{\Sigma \Gamma}-K_{\Gamma \Sigma}^{h} K_{\Sigma \Gamma}^{h}=K_{\Gamma \Sigma}\left(K_{\Sigma \Gamma}-K_{\Sigma \Gamma}^{h}\right)+\left(K_{\Gamma \Sigma}-K_{\Gamma \Sigma}^{h}\right) K_{\Sigma \Gamma}^{h} .
$$

It is clear that the first addendum converges to zero by (a). It is easy to prove that $K_{\Sigma \Gamma}^{h}$ : $H^{1 / 2}(\Gamma) \rightarrow H^{1}(\Sigma)$ is uniformly bounded (see Proposition 8.8). The family $\left\{K_{\Sigma \Gamma}^{h}\right\}$ is therefore collectively compact (see [12, Ch. 10]). Then (b) and the collective compactness of this family ensure (see [12, Theorem 10.7]) uniform convergence of the second term in (12).

Corollary 4.1. If $V_{\Gamma}$ is elliptic, $\rho(\mathcal{K})<1$, and Hypothesis 1 holds, then for $h$ small enough, $\rho\left(K_{\Gamma \Sigma}^{h} K_{\Sigma \Gamma}^{h}\right) \leqslant \rho_{0}<1$.

Proof. In $H^{1 / 2}(\Gamma)$ we consider the equivalent norm $\left\langle V_{\Gamma}^{-1} \cdot, \cdot\right\rangle^{1 / 2}$ and the corresponding operator norm $\||\cdot|\|$

$$
\|\| B \|:=\sup _{0 \neq g \in H^{-1 / 2}(\Gamma)} \frac{\left\langle V_{\Gamma}^{-1} B g, B g\right\rangle^{1 / 2}}{\left\langle V_{\Gamma}^{-1} g, g\right\rangle^{1 / 2}}
$$

in the set of bounded linear maps from $H^{1 / 2}(\Gamma)$ into itself, equivalent to the common one. Taking the logical definitions for $V_{\Sigma}$ and $V_{\Gamma \Sigma}$, which is the transposed of $V_{\Sigma \Gamma}$, we have that $K_{\Gamma \Sigma} K_{\Sigma \Gamma}=V_{\Gamma \Sigma} V_{\Sigma}^{-1} V_{\Sigma \Gamma} V_{\Gamma}^{-1}$ is compact and selfadjoint in this new inner product (see [3] Theorem 3.2 for the necessary adjustments if $V_{\Sigma}$ is not inversible). Therefore it follows that

$$
\rho\left(K_{\Gamma \Sigma} K_{\Sigma \Gamma}\right)=\left\|\left|K_{\Gamma \Sigma} K_{\Sigma \Gamma} \|\right|<1 .\right.
$$

Hence

$$
\rho\left(K_{\Gamma \Sigma}^{h} K_{\Sigma \Gamma}^{h}\right) \leqslant\left\|\left|K _ { \Gamma \Sigma } ^ { h } K _ { \Sigma \Gamma } ^ { h } \left\|\left|\leqslant\left\|\left|K _ { \Gamma \Sigma } K _ { \Sigma \Gamma } \left\|\left|+\left\|\left|K_{\Gamma \Sigma}^{h} K_{\Sigma \Gamma}^{h}-K_{\Gamma \Sigma} K_{\Sigma \Gamma}\|\rightarrow\|\left\|K_{\Gamma \Sigma} K_{\Sigma \Gamma}\right\|\right| .\right.\right.\right.\right.\right.\right.\right.\right.\right.
$$


Theorem 4.2. If $V_{\Gamma}$ is elliptic, $\rho(\mathcal{K})<1$, and Hypothesis 1 holds, then $\mathcal{A}_{h}$ is inversible for $h$ small enough and the iterative process converges with an $h$-independent velocity of convergence.

Proof. As a consequence of the preceding result, for $h$ small enough

$$
\rho\left(\mathcal{K}_{h}\right)^{2}=\rho\left(K_{\Gamma \Sigma}^{h} K_{\Sigma \Gamma}^{h}\right) \leqslant \rho_{0}<1 .
$$

This inequality proves the result.

Theorem 4.3. If $V_{\Gamma}$ is elliptic, $\rho(\mathcal{K})<1$, and Hypothesis 1 holds, then the inverse of $\mathcal{A}_{h}$ is uniformly bounded for $h$ small enough.

Proof. We consider in $H^{1 / 2}(\Gamma)$ the non-standard norm $\left\langle V_{\Gamma}^{-1} \cdot, \cdot\right\rangle^{1 / 2}$ defined in the proof of Corollary 4.1 and the associated operator norm (13). Since $\left\|\left|K_{\Gamma \Sigma}^{h} K_{\Sigma \Gamma}^{h} \|\right| \leqslant \tau<1\right.$, with $\tau$ independent of $h$, it is clear that

$$
\left\|\left(K_{\Sigma \Gamma}^{h} K_{\Gamma \Sigma}^{h}\right)^{N}\right\| \leqslant\left\|K_{\Sigma \Gamma}^{h}\right\|\left\|\left(K_{\Gamma \Sigma}^{h} K_{\Sigma \Gamma}^{h}\right)^{N-1}\right\|\left\|K_{\Gamma \Sigma}^{h}\right\| \leqslant C\|\| K_{\Gamma \Sigma}^{h} K_{\Sigma \Gamma}^{h}\|\|^{N-1} \leqslant C \tau^{N-1} .
$$

Taking $N$ large enough, we obtain $\left\|\mathcal{K}_{h}^{2 N}\right\| \leqslant \tau^{\prime}<1$, which implies the result.

\section{Overlapped finite and boundary elements}

Our next step is to show that Theorem 4.3 can be proven without assuming that $\rho(\mathcal{K})<1$, but assuming only inversibility of $V_{\Gamma}$, i.e., of $\mathcal{A}$. This can be accomplished by assuming an additional approximation property for $S_{h}^{\Gamma}$. The effect on the analysis will be the fact that we will be able to consider the discrete method (8) as an approximation scheme in itself, not only as the limit of the Schwarz method. This method is then the overlapping of the finite element and boundary element discretization. Since the restriction of $\mathcal{A}_{h}$ to $X_{h}^{\Sigma} \times X_{h}^{\Gamma}$ has images on this same space, in practice equations (8) can be understood in this discrete space. Therefore $N_{h}$ is never used in implementation. Besides, we can always revert to the equivalent formulation (5).

The following hypothesis implies Hypothesis 1.

Hypothesis 2. There exists $\alpha$ such that $\alpha(h) \rightarrow 0$ as $h \rightarrow 0$ and

$$
\inf _{\psi_{h} \in S_{h}^{\Gamma}}\left\|\psi-\psi_{h}\right\|_{-1 / 2, \Gamma} \leqslant \alpha(h)\|\psi\|_{0, \Gamma}, \quad \forall \psi \in H^{0}(\Gamma) .
$$

Proposition 5.1. If Hypothesis 2 holds, then there exists $\beta$ such that $\beta(h) \rightarrow 0$ as $h \rightarrow 0$ and

$$
\left\|L_{h} f-f\right\|_{0, \Gamma} \leqslant \beta(h)\|f\|_{1 / 2, \Gamma}, \quad \forall f \in H^{1 / 2}(\Gamma) .
$$

Proof. Let $\psi:=V_{\Gamma}^{-1} f$ and $\psi_{h}=V_{\Gamma}^{-1} L_{h} f$. Then $\left\|L_{h} f-f\right\|_{0, \Gamma} \leqslant C\left\|\psi-\psi_{h}\right\|_{-1, \Gamma}$, since $V_{\Gamma}: H^{-1}(\Gamma) \rightarrow H^{0}(\Gamma)$ is bounded (see [7]). The result is then a simple consequence of the use of Aubin-Nitsche type estimates (see [16, Ch. 1]).

Let $N_{h}: H^{1 / 2}(\Sigma) \rightarrow X_{h}^{\Sigma} \subset H^{1 / 2}(\Sigma)$ be now any projection onto $X_{h}^{\Sigma}$ such that it is $h$-uniformly bounded and

$$
\left\|N_{h} g-g\right\|_{0, \Sigma} \leqslant \delta(h)\|g\|_{1 / 2, \Sigma}, \quad \forall g \in H^{1 / 2}(\Sigma), \quad \lim _{h \rightarrow 0} \delta(h)=0 .
$$

The existence of such a projection is guaranteed by Proposition 8.6. Moreover, if we renew the definition of $K_{\Gamma \Sigma}^{h}$, then Propositions 3.3, 4.1, 4.2 and Theorem 4.1 still hold. 
Theorem 5.1. If Hypothesis 2 holds, then

$$
K_{\Sigma \Gamma}^{h} K_{\Gamma \Sigma}^{h} \rightarrow K_{\Sigma \Gamma} K_{\Gamma \Sigma}
$$

Hence $\mathcal{K}_{h}^{2} \rightarrow \mathcal{K}^{2}$

Proof. Since by Theorem 4.1(a) we have that $K_{\Sigma \Gamma}^{h} \rightarrow K_{\Sigma \Gamma}$, we just have to prove that $K_{\Sigma \Gamma} K_{\Gamma \Sigma}^{h} \rightarrow K_{\Sigma \Gamma} K_{\Gamma \Sigma}$. Since $K_{\Sigma \Gamma}: H^{0}(\Gamma) \rightarrow H^{1 / 2}(\Sigma)$ is bounded, we can simply prove that

$$
\left\|\left(K_{\Gamma \Sigma}^{h}-K_{\Gamma \Sigma}\right) g\right\|_{0, \Gamma} \leqslant \varepsilon(h)\|g\|_{1 / 2, \Sigma}
$$

with $\varepsilon(h) \rightarrow 0$. By the definitions of the operators involved

$$
\left\|\left(K_{\Gamma \Sigma}^{h}-K_{\Gamma \Sigma}\right) g\right\|_{0, \Gamma} \leqslant\left\|\left(L_{h}-I\right) \gamma_{\Gamma} R_{h} N_{h} g\right\|_{0, \Gamma}+\left\|\gamma_{\Gamma}\left(R_{h} N_{h}-R\right) g\right\|_{0, \Gamma} .
$$

For the first term we use Proposition 5.1 and the uniform boundedness of $R_{h}$ (see Proposition 8.4)

$$
\left\|\left(L_{h}-I\right) \gamma_{\Gamma} R_{h} N_{h} g\right\|_{0, \Gamma} \leqslant \beta(h)\left\|\gamma_{\Gamma} R_{h} N_{h} g\right\|_{1 / 2, \Gamma} \leqslant \beta(h) C\|g\|_{1 / 2, \Sigma} .
$$

On the other hand, for arbitrary $\eta \in(0,1 / 2)$

$$
\begin{aligned}
\left\|\gamma_{\Gamma}\left(R_{h} N_{h}-R\right) g\right\|_{0, \Gamma} & \leqslant C\left\|\left(R_{h} N_{h}-R\right) g\right\|_{1 / 2+\eta, Q} \\
& \leqslant C\left\|\left(R_{h}-R\right) N_{h} g\right\|_{1 / 2+\eta, Q}+C\left\|N_{h} g-g\right\|_{\eta, \Sigma}
\end{aligned}
$$

where we have applied Propositions 8.1 and 8.2. From the interpolation properties of Sobolev spaces, Proposition 8.5 and Proposition 8.4 (with $s=0$ ) we have

$$
\begin{aligned}
\left\|\left(R_{h}-R\right) N_{h} g\right\|_{1 / 2+\eta, Q} & \leqslant\left\|\left(R_{h}-R\right) N_{h} g\right\|_{0, Q}^{1 / 2-\eta}\left\|\left(R_{h}-R\right) N_{h} g\right\|_{1, Q}^{1 / 2+\eta} \\
& \leqslant \gamma(h)^{1 / 2-\eta} C\left\|N_{h} g\right\|_{1 / 2, \Sigma} .
\end{aligned}
$$

The remaining bound follows again by interpolation of Sobolev spaces and (14)

$$
\left\|N_{h} g-g\right\|_{\eta, \Sigma} \leqslant\left\|N_{h} g-g\right\|_{0, \Sigma}^{1-2 \eta}\left\|N_{h} g-g\right\|_{1 / 2, \Sigma}^{2 \eta} \leqslant \delta(h)^{1-2 \eta} C\|g\|_{1 / 2, \Sigma}
$$

Gathering (16) - (20), we prove (15) and thus the first result. The second one is a simple consequence of this and Theorem 4.1(d).

Theorem 5.2. If $V_{\Gamma}^{-1}$ exists and Hypothesis 2 holds, then $\mathcal{A}_{h}$ is inversible for $h$ small enough and its inverse is $h$-uniformly bounded.

Proof. Let $\mathcal{B}:=\mathcal{I}-\mathcal{K}$ and $\mathcal{B}_{h}:=\mathcal{I}-\mathcal{K}_{h}$. From the particular matrix form of $\mathcal{K}$, it is clear that its spectrum is symmetric about the origin. Hence, $\mathcal{B}$ is one-to-one. By the Fredholm alternative, this implies that $\mathcal{B}$ is an isomorphism. Moreover,

$$
\mathcal{A}_{h} \mathcal{B}_{h}=\left(\mathcal{I}+\mathcal{K}_{h}\right)\left(\mathcal{I}-\mathcal{K}_{h}\right)=\mathcal{I}-\mathcal{K}_{h}^{2} \rightarrow \mathcal{I}-\mathcal{K}^{2}=\mathcal{A B}
$$

and therefore $\mathcal{A}_{h} \mathcal{B}_{h}$ is inversible for $h$ small enough with a uniformly bounded inverse. The family of operators $\mathcal{C}_{h}:=\mathcal{B}_{h}\left(\mathcal{A}_{h} \mathcal{B}_{h}\right)^{-1}$ is uniformly bounded and $\mathcal{A}_{h} \mathcal{C}_{h}=\mathcal{I}$. This implies, by the Fredholm alternative, that $\mathcal{A}_{h}$ is inversible and hence $\mathcal{C}_{h}=\mathcal{A}_{h}^{-1}$. 


\section{Approximation properties}

In this section, we perform an asymptotic analysis of the approximation of the solution of (7) by that of (8) valid in the hypotheses of the preceding sections. We will actually use the fact that $\mathcal{A}_{h}$ has a uniformly bounded inverse (Theorems 4.3 and 5.2).

We simply recall that $g_{0, h}^{\Sigma} \approx g_{\Sigma}$ is the initial choice, but $g_{0, h}^{\Gamma}=V_{\Gamma} \operatorname{BEM}\left(g_{\Gamma}\right)$ is the image by $V_{\Gamma}$ of the density calculated by the boundary element method for the data on $\Gamma$.

If $\left(f_{\Sigma}, f_{\Gamma}\right)$ solves $(7)$, then the solution $w \in H^{1}(\Omega)$ to $(1)$ is decomposed as

$$
w=u+\mathcal{S}_{\Gamma} \psi, \quad u:=R f_{\Sigma}, \quad \psi:=V_{\Gamma}^{-1} f_{\Gamma} .
$$

The discrete version of this is

$$
w_{h}:=u_{h}+\mathcal{S}_{\Gamma} \psi_{h}, \quad u_{h}:=R_{h} f_{h}^{\Sigma}, \quad \psi_{h}:=V_{\Gamma}^{-1} f_{h}^{\Gamma},
$$

$\left(f_{h}^{\Sigma}, f_{h}^{\Gamma}\right)$ being the solution of (8). Therefore, $\left(u_{h}, \psi_{h}\right)$ is the solution to (5).

Proposition 6.1. If $g_{0, h}^{\Sigma} \rightarrow g_{\Sigma}$ in $H^{1 / 2}(\Sigma)$ as $h \rightarrow 0$, then

$$
\lim _{h \rightarrow 0}\left(\left\|f_{h}^{\Sigma}-f_{\Sigma}\right\|_{1 / 2, \Sigma}+\left\|f_{h}^{\Gamma}-f_{\Gamma}\right\|_{1 / 2, \Gamma}\right)=0
$$

and therefore

$$
\lim _{h \rightarrow 0}\left\|w-\left(u_{h}+\mathcal{S}_{\Gamma} \psi_{h}\right)\right\|_{1, \Omega}=0
$$

Proof. Notice that $g_{0, h}^{\Gamma} \rightarrow g_{\Gamma}$ in $H^{1 / 2}(\Gamma)$ (see Hypothesis 1 ). Since $\mathcal{A}_{h} \stackrel{\mathrm{pt}}{\rightarrow} \mathcal{A}$ and $\mathcal{A}_{h}^{-1}$ is uniformly bounded, it follows that $\mathcal{A}_{h}^{-1} \stackrel{\mathrm{pt}}{\rightarrow} \mathcal{A}^{-1}$, which proves (22). Then (23) is a simple consequence of the boundedness of $\mathcal{S}_{\Gamma} V_{\Gamma}^{-1}: H^{1 / 2}(\Gamma) \rightarrow H^{1}(\Omega)$ and of Propositions 4.2 and 8.4 .

Theorem 6.1. In the notations above,

$$
\begin{aligned}
\left\|w-w_{h}\right\|_{1, \Omega} \leqslant C[ & \left\|g_{\Sigma}-g_{0, h}^{\Sigma}\right\|_{1 / 2, \Sigma}+\inf _{\psi_{h} \in S_{h}^{\Gamma}}\left\|V_{\Gamma}^{-1} g_{\Gamma}-\psi_{h}\right\|_{-1 / 2, \Gamma} \\
& \left.+\inf _{p_{h} \in P_{h}^{Q}}\left\|u-p_{h}\right\|_{1, Q}+\inf _{\psi_{h} \in S_{h}^{\Gamma}}\left\|\psi-\psi_{h}\right\|_{-1 / 2, \Gamma}+h^{k+1 / 2}\|\psi\|_{-1 / 2, \Gamma}\right] .
\end{aligned}
$$

Proof. Since $R_{h} N_{h} \gamma_{\Sigma} u_{h}=u_{h}$, it readily follows that

$$
\begin{aligned}
\left\|u-u_{h}\right\|_{1, Q} & \leqslant\left\|u-R_{h} N_{h} \gamma_{\Sigma} u\right\|_{1, Q}+\left\|R_{h} N_{h}\left(\gamma_{\Sigma} u-\gamma_{\Sigma} u_{h}\right)\right\|_{1, Q} \\
& \leqslant C\left[\inf _{p_{h} \in P_{h}^{Q}}\left\|u-p_{h}\right\|_{1, Q}+\left\|f_{\Sigma}-f_{h}^{\Sigma}\right\|_{1 / 2, \Sigma}\right]
\end{aligned}
$$

where we have applied Proposition 8.7 and the uniform boundedness of $R_{h}$ and $N_{h}$. It is also clear that

$$
\left\|\mathcal{S}_{\Gamma} \psi-\mathcal{S}_{\Gamma} \psi_{h}\right\|_{1, \Omega} \leqslant C\left\|f_{\Gamma}-f_{h}^{\Gamma}\right\|_{1 / 2, \Gamma}
$$

If we use the uniform boundedness of $\mathcal{A}_{h}^{-1}$ and the identities

$$
\mathcal{A}_{h}\left[\begin{array}{c}
f_{\Sigma}-f_{h}^{\Sigma} \\
f_{\Gamma}-f_{h}^{\Gamma}
\end{array}\right]=\left[\begin{array}{c}
g_{\Sigma} \\
g_{\Gamma}
\end{array}\right]-\left[\begin{array}{c}
g_{0, h}^{\Sigma} \\
g_{0, h}^{\Gamma}
\end{array}\right]+\left(\mathcal{K}_{h}-\mathcal{K}\right)\left[\begin{array}{c}
f_{\Sigma} \\
f_{\Gamma}
\end{array}\right]=\left[\begin{array}{c}
g_{\Sigma}-g_{0, h}^{\Sigma} \\
g_{\Gamma}-L_{h} g_{\Gamma}
\end{array}\right]+\left[\begin{array}{c}
\left(K_{\Sigma \Gamma}^{h}-K_{\Sigma \Gamma}\right) f_{\Gamma} \\
\left(K_{\Gamma \Sigma}^{h}-K_{\Gamma \Sigma}\right) f_{\Sigma}
\end{array}\right]
$$


and

$$
\left(K_{\Gamma \Sigma}^{h}-K_{\Gamma \Sigma}\right) f_{\Sigma}=\left(L_{h}-I\right)\left(g_{\Gamma}-f_{\Gamma}\right)+L_{h} \gamma_{\Gamma}\left(R_{h} N_{h}-R\right) f_{\Sigma}
$$

it follows that

$$
\begin{aligned}
\left\|f_{\Sigma}-f_{h}^{\Sigma}\right\|_{1 / 2, \Sigma}+\left\|f_{\Gamma}-f_{h}^{\Gamma}\right\|_{1 / 2, \Gamma} \leqslant & C\left[\left\|g_{\Sigma}-g_{0, h}^{\Sigma}\right\|_{1 / 2, \Sigma}+\left\|g_{\Gamma}-L_{h} g_{\Gamma}\right\|_{1 / 2, \Gamma}\right. \\
& +\left\|\left(I_{h}^{\Sigma}-I\right) \gamma_{\Sigma} \mathcal{S}_{\Gamma} \psi\right\|_{1 / 2, \Sigma}+\left\|f_{\Gamma}-L_{h} f_{\Gamma}\right\|_{1 / 2, \Gamma} \\
& \left.+\left\|L_{h} \gamma_{\Gamma}\left(R_{h} N_{h}-R\right) f_{\Sigma}\right\|_{1 / 2, \Gamma}\right] .
\end{aligned}
$$

Since $L_{h}$ is a uniformly bounded projection onto $X_{h}^{\Gamma}=V_{\Gamma} S_{h}^{\Gamma}$, we obtain

$$
\left\|g_{\Gamma}-L_{h} g_{\Gamma}\right\|_{1 / 2, \Gamma} \leqslant C \inf _{r_{h} \in X_{h}^{\Gamma}}\left\|g_{\Gamma}-r_{h}\right\|_{1 / 2, \Gamma} \leqslant C^{\prime} \inf _{\psi_{h} \in S_{h}^{\Gamma}}\left\|V_{\Gamma}^{-1} g_{\Gamma}-\psi_{h}\right\|_{-1 / 2, \Gamma}
$$

The fourth term in (26) is bounded similarly. For the third term we apply Proposition 8.8 and for the fifth one the uniform boundedness of $L_{h}$ and Proposition 8.7. Gathering these bounds, (24), (25) and (26), the result follows readily.

Proposition 6.2. If the solution of (1), $w$, is decomposed as in (21), then for $s>1$, $w \in H^{s}(\Omega)$ if and only if $u \in H^{s}(Q)$ and $\mathcal{S}_{\Gamma} \psi \in H^{s}(\Omega)$.

Proof. It is a simple consequence of the fact that $u \in \mathcal{C}^{\infty}(Q)$ and $\mathcal{S}_{\Gamma} \psi \in \mathcal{C}^{\infty}\left(\mathbb{R}^{d} \backslash \Gamma\right)$.

Finite element error. Because of Theorem 6.1, we can now study the discretization error as a sum of different effects, part of them depending on the finite element discretization and the remaining-on the boundary elements. We now denote $h_{Q}$ to the maximum diameter in the triangulation. Assuming that $w \in H^{k+1}(\Omega)$ and taking $g_{0, h}^{\Sigma}:=I_{h}^{\Sigma} g_{\Sigma}$, we obtain that

$$
\inf _{p_{h} \in P_{h}^{Q}}\left\|u-p_{h}\right\|_{1, Q} \leqslant C h_{Q}^{k}|u|_{k+1, Q}
$$

and

$$
\left\|g_{\Sigma}-g_{h, 0}^{\Sigma}\right\|_{1 / 2, \Sigma} \leqslant C h_{Q}^{k}\|g\|_{k+1 / 2, \Sigma^{*}}
$$

where the norm of $g$ in the right hand side of the last expression is taken as the sum of the norms of each side/face of $\Sigma$.

Notice that this last bound can be put in reference to the diameter of the inherited triangulation of $\Sigma$ instead of the interior diameter $h_{Q}$. The fifth addendum of the error in Theorem 6.1 uses this same quantity, because it proceeds from the boundary interpolation error.

Boundary element error. If $\Gamma$ is a smooth boundary (let us assume it to be $\mathcal{C}^{\infty}$ for simplicity), then $w \in H^{k+1}(\Omega)$ implies that $\psi$ and $V_{\Gamma}^{-1} g_{\Gamma}$ belong to $H^{k-1 / 2}(\Gamma)$. We can take $S_{h}^{\Gamma}$ to be a space of piecewise polynomials of degree $k-1$ (not necessarily continuous). Hypotheses 1 and 2 are then trivially satisfied.

Then, the terms related to the boundary discretization provide an error behaving like $\mathcal{O}\left(h_{\Gamma}^{k}\right)$ (see $\left.[5,21]\right)$. In fact, by assuming some further regularity, namely $w \in H^{k+3 / 2}(\Omega)$, this bound can be improved by one half.

Although difficult to implement, trigonometric polynomials/spherical harmonics provide very good error bounds. If $\Gamma$ is composed of several disconnected smooth closed 
curves/surfaces, diffeomorphic to circumferences/spheres, and we have parameterizations of them, then we can use this kind of spectral approximation. If the data on $\Gamma$ satisfy $g_{\Gamma} \in \mathcal{C}^{\infty}(\Gamma)$, then $f_{\Gamma}=g_{\Gamma}-K_{\Gamma \Sigma} f_{\Sigma} \in \mathcal{C}^{\infty}(\Gamma)$ and we can bound both error terms by $C_{m} N^{-m}, N$ being the number of degrees of freedom on $\Gamma$ and $m$ any positive integer. The bounds can be improved to give exponential order with additional analyticity assumptions. This means that very few degrees of freedom are needed in $\Gamma$. For more on spectral boundary element discretizations in two dimensions see [18] (see also [15] for spherical harmonics in relationship with Sobolev spaces).

\section{Some numerical experiments}

We illustrate some of the foregoing results by means of a simple two-dimensional example. The complete domain $Q$ is the rectangle $[0,4] \times[0,1]$. The obstacles are three circles with centers on $(2,0.75),(1.25,0.4)$ and $(2.75,0.4)$ and respective radii $0.12,0.09,0.09$. Their boundaries are denoted by $\Gamma_{k}(k=1,2,3)$. We take the Dirichlet data so that the exact solution of (1) is

$$
\begin{aligned}
w(x, y)= & (x-2)^{4}-6 y^{2}(x-2)^{2}+y^{4} \\
& \left.+\log \left((x-2.05)^{2}+(y-0.7)^{2}\right)+\log \left((x-1.3)^{2}\right)+(y-0.42)^{2}\right) .
\end{aligned}
$$

Decomposition (21) of $w$ that the algorithm finds takes $u(x, y)=(x-2)^{4}-6 y^{2}(x-2)^{2}+y^{4}$.

In $Q$ we define a uniform triangular mesh based on taking $N$ equal partitions on the vertical side and $4 N$ on the horizontal direction and continuous $\mathbb{P}_{1}$ elements. Notice that the stiffness matrix of the FEM procedure is (up to a multiplicative factor) the same as that of the central finite difference method on the uniform grid formed by the vertices of all triangles (see [10]). The trace on the exterior boundary $g_{0, h}^{\Sigma}$ is that given by linear interpolation. We profit from the simple structure of the FE problem and from the values of $N$ that will be chosen to use the FFT technique to solve the corresponding linear systems (see [14, Ch. 4]).

The single layer potential is taken in a parameterized form. Let $\left(c_{x}^{k}, c_{y}^{k}\right)$ be the center of $\Gamma_{k}, r_{k}$ its radius and $\gamma_{k}(t):=\left(c_{x}^{k}, c_{y}^{k}\right)+r_{k}(\cos 2 \pi t, \sin 2 \pi t)$ a parametrization of $\Gamma_{k}$. We take

$$
\sum_{k=1}^{3} \int_{0}^{1} \log \left|x-\gamma_{k}(t)\right| \psi_{k}(t) \mathrm{d} t
$$

as a solution of the exterior problem with densities $\psi_{k}$ to be determined.

The BEM procedure we consider here is a non-conforming Petrov-Galerkin delta-delta method, which does not fit in the hypotheses but has the advantage of its great simplicity. The method appears as a quadrature method in [17] and is also derived in a very different setting in [4]. We take $M$ equally spaced points on the interval used for parameterization of each curve: $t_{i}:=i h(i=1, \ldots, M, h:=1 / M)$ and also the displaced grid $s_{i}:=(i+1 / 6) h$. With the right hand side function $g: \Gamma_{1} \cup \Gamma_{2} \cup \Gamma_{3} \rightarrow \mathbb{R}$ we have to solve a $3 M \times 3 M$ system given by the equations

$$
\sum_{k=1}^{3} \sum_{j=1}^{M} \log \left|\gamma_{\ell}\left(s_{i}\right)-\gamma_{k}\left(t_{j}\right)\right| \psi_{k, j}=g\left(\gamma_{\ell}\left(s_{i}\right)\right), \quad \ell=1 \ldots, 3 ; i=1, \ldots, M .
$$


The corresponding potential is then

$$
\sum_{k=1}^{3} \sum_{j=1}^{M} \log \left|x-\gamma_{k}\left(t_{j}\right)\right| \psi_{k, j},
$$

which has singularities on the points $\gamma_{k}\left(t_{j}\right) \in \Gamma_{k}$. These singularities provoke a loss in the order of convergence when we approach the curves, but do not affect the convergence away from the obstacles.

In Figure 2 we show the contour lines of the interior solution $u$, the exterior solution $\mathcal{S}_{\Gamma} \psi$ and their sum computed with $N=32$ and $M=40$.
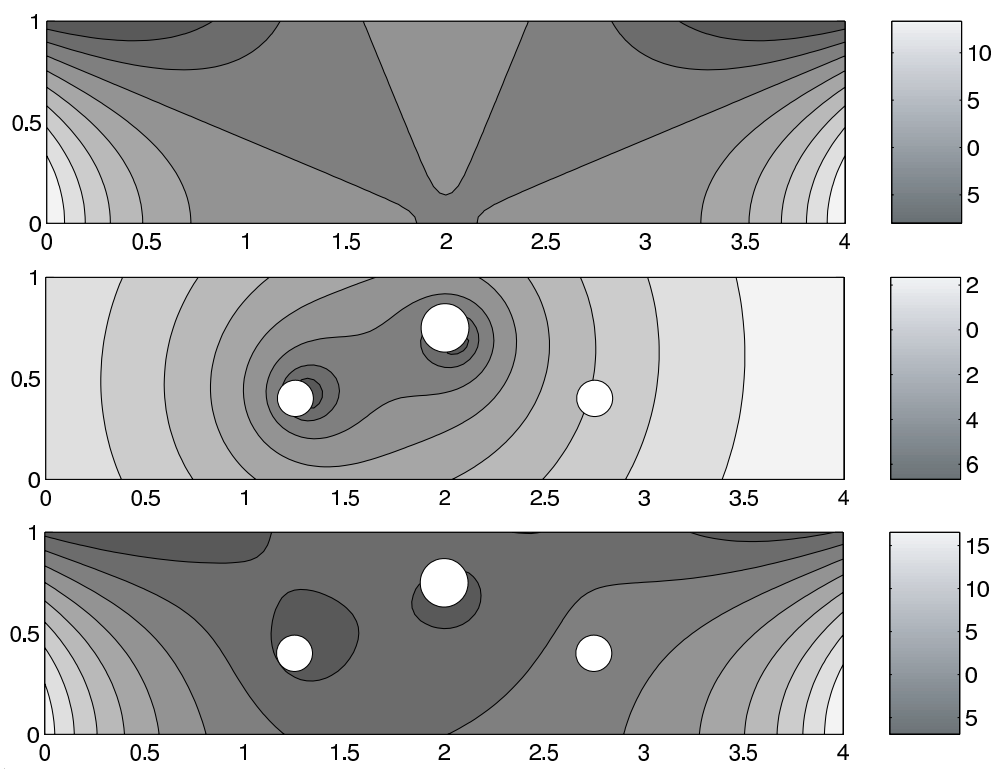

Figure 2. Interior, exterior and full solution of the problem

For different values of $N$ and $M$, we apply the Jacobi-Schwarz iteration (4) and also GMRES to the equivalent system (5). Convergence of the exact Schwarz iteration is ensured by [3], Proposition 3.3. The stopping criterion for both iterations is based on the relative error of the residual. Table 1 shows the number of Schwarz and GMRES iterations. Notice that the number of iterations is independent of $N$ and $M$. The spectral radius of $\mathcal{K}_{h}$ is approximately 0.66. Table 1 also shows the maximum error of the full solution on the nodes placed on $\Omega$ and also the maximum nodal error in nodes such that their distance with the interior boundaries is bigger than 0.25, where the attained convergence order is clearly 2 .

\section{Appendix}

In this section, we gather some results that were use in the preceding work and give them in the precise form needed. The domain $Q$ considered here has a polygonal/polyhedral boundary $\Sigma(Q$ may not be simply connected) and the corresponding harmonic lifting is denoted by $R$ (see (6)). At the discrete level, we consider the finite element space $P_{h}^{Q}$ of Lagrangian simplices of degree $k$, its trace $X_{h}^{\Sigma}=\gamma_{\Sigma} P_{h}^{Q}$, and the discrete harmonic lifting $R_{h}$ (see (3)). 
Table 1. Number of iterations and errors

\begin{tabular}{|c|c|c|c|c|c|}
\hline$N$ & $M$ & \# Schwarz it. & \# GMRES it. & Nodal error & Nodal error away from $\Gamma$ \\
\hline 8 & 10 & 25 & 10 & $2.80 \mathrm{E}-1$ & $1.44 \mathrm{E}-2$ \\
16 & 20 & 25 & 10 & $8.39 \mathrm{E}-2$ & $3.96 \mathrm{E}-3$ \\
32 & 40 & 25 & 10 & $2.83 \mathrm{E}-2$ & $1.03 \mathrm{E}-3$ \\
64 & 80 & 25 & 10 & $3.76 \mathrm{E}-3$ & $2.63 \mathrm{E}-4$ \\
\hline
\end{tabular}

Proposition $8.1[7,13]$. The trace operator $\gamma_{\Sigma}: H^{s+1 / 2}(Q) \rightarrow H^{s}(\Sigma)$ is well defined and bounded for all $s \in(0,1)$.

Obviously, this result also holds for the interior trace on $\Gamma$.

Proposition $8.2[7,13]$. The harmonic lifting $R: H^{s}(\Sigma) \rightarrow H^{s+1 / 2}(Q)$ is well defined and bounded for all $s \in[0,1]$.

The following result refers to the Scott-Zhang projection onto finite element spaces. The exact definition of this operator is immaterial for our needs; only some of its properties are used.

Proposition 8.3 [19]. There exists a linear projection $\Pi_{h}: H^{1}(Q) \rightarrow P_{h}^{Q}$ such that:

(a) $\Pi_{h} u \in H_{0}^{1}(Q)$ for all $u \in H_{0}^{1}(Q)$.

(b) For all $0 \leqslant s \leqslant 1 \leqslant t \leqslant d+1$, there exists a constant $C$ independent of $h$ such that

$$
\left\|\Pi_{h} u-u\right\|_{s, Q} \leqslant C h^{t-s}|u|_{t, Q}, \quad \forall u \in H^{t}(Q)
$$

Proposition 8.4. For all $s \in[0,1 / 2]$, there exists $C$ independent of $h$ such that

$$
\left\|R g_{h}-R_{h} g_{h}\right\|_{1, Q} \leqslant C h^{s}\left\|g_{h}\right\|_{1 / 2+s, \Sigma}, \quad \forall g_{h} \in X_{h}^{\Sigma}
$$

Proof. Since $R g_{h}-R_{h} g_{h} \in H_{0}^{1}(Q)$ and $\Pi_{h}$ is a projection, $\Pi_{h} R g_{h}-R_{h} g_{h} \in H_{0}^{1}(Q) \cap P_{h}^{Q}$ and thus

$$
\begin{aligned}
\int_{Q} \nabla\left(R-R_{h}\right) g_{h} \cdot \nabla\left(R-R_{h}\right) g_{h} & =\int_{Q} \nabla\left(R-R_{h}\right) g_{h} \cdot \nabla\left(R-\Pi_{h} R\right) g_{h} \\
& \leqslant\left|\left(R-R_{h}\right) g_{h}\right|_{1, Q}\left|\left(R-\Pi_{h} R\right) g_{h}\right|_{1, Q} .
\end{aligned}
$$

On the other hand, the preceding results show, that

$$
\left\|R g_{h}-\Pi_{h} R g_{h}\right\|_{1, Q} \leqslant C h^{s}\left\|R g_{h}\right\|_{1+s, Q} \leqslant C^{\prime} h^{s}\left\|g_{h}\right\|_{1 / 2+s, \Sigma}
$$

holds for all $g_{h} \in X_{h}^{\Sigma}$ and $s \in[0,1 / 2]$. The result then follows from (27), (28) and the Poincaré inequality.

Proposition 8.5. There exists a function $\gamma$ such that $\gamma(h) \rightarrow 0$ as $h \rightarrow 0$ and

$$
\left\|R g_{h}-R_{h} g_{h}\right\|_{0, Q} \leqslant \gamma(h)\left\|g_{h}\right\|_{1 / 2, \Sigma}, \quad \forall g_{h} \in X_{h}^{\Sigma}
$$


Proof. The quantity we want to estimate can be written

$$
\left\|R g_{h}-R_{h} g_{h}\right\|_{0, Q}=\sup _{0 \neq \phi \in L^{2}(Q)} \frac{1}{\|\phi\|_{0, Q}} \int_{Q}\left(R g_{h}-R_{h} g_{h}\right) \phi .
$$

For a given $\phi \in L^{2}(Q)$, we take the unique $w \in H_{0}^{1}(Q)$ such that $\Delta w=\phi$. With the regularity assumed for the boundary we can estimate (see [9])

$$
\|w\|_{3 / 2, Q} \leqslant C\|\phi\|_{0, Q}
$$

with $C$ independent of $\phi$. Since $\Pi_{h} w \in H_{0}^{1}(Q) \cap P_{h}^{Q}$, it follows that

$$
\begin{aligned}
\int_{Q}\left(R g_{h}-R_{h} g_{h}\right) \Delta w & =\int_{Q} \nabla\left(R g_{h}-R_{h} g_{h}\right) \cdot \nabla w=\int_{Q} \nabla\left(R g_{h}-R_{h} g_{h}\right) \cdot \nabla\left(w-\Pi_{h} w\right) \\
& \leqslant\left\|R g_{h}-R_{h} g_{h}\right\|_{1, Q}\left\|w-\Pi_{h} w\right\|_{1, Q} \leqslant C h^{1 / 2}\left\|g_{h}\right\|_{1 / 2, Q}\|w\|_{3 / 2, Q}
\end{aligned}
$$

according to Propositions 8.3 and 8.4. This inequality, (29), and (30) prove the result.

Bound (30) is not optimal in the sense that some higher norm of $w$ can be estimated. This would allow for some better value for the function $\gamma(h)$, which is however irrelevant for our purposes.

Proposition 8.6. The operator $N_{h}:=\gamma_{\Sigma} \Pi_{h} R$ satisfies:

(a) $N_{h}^{2}=N_{h}$.

(b) There exists $C$ independent of $h$ such that

$$
\left\|N_{h} g\right\|_{1 / 2, \Sigma} \leqslant C\|g\|_{1 / 2, \Sigma}, \quad \forall g \in H^{1 / 2}(\Sigma) .
$$

(c) There exists a function $\delta$ such that $\delta(h) \rightarrow 0$ as $h \rightarrow 0$ and

$$
\left\|N_{h} g-g\right\|_{0, \Sigma} \leqslant \delta(h)\|g\|_{1 / 2, \Sigma}, \quad \forall g \in H^{1 / 2}(\Sigma) .
$$

Proof. Both (a) and (b) are straightforward, using Proposition 8.3 with $s=t=1$ for this last. From Propositions 8.1 and 8.3 it follows that for all $\varepsilon \in(0,1 / 2]$

$$
\left\|N_{h} g-g\right\|_{0, \Sigma}=\left\|\gamma_{\Sigma}\left(\Pi_{h}-I\right) R g\right\|_{0, \Sigma} \leqslant C h^{1 / 2-\varepsilon}\|R g\|_{1, Q} \leqslant C^{\prime} h^{1 / 2-\varepsilon}\|g\|_{1 / 2, \Sigma}
$$

for all $g \in H^{1 / 2}(\Sigma)$.

Proposition 8.7. Let $N_{h}: H^{1 / 2}(\Sigma) \rightarrow X_{h}^{\Sigma}$ satisfy the properties (a) and (b) of Proposition 8.6. Then there exists $C$ independent of $h$ such that for all $u \in H^{1}(Q)$ with $\Delta u=0$ in $Q$,

$$
\left\|u-R_{h} N_{h} \gamma_{\Sigma} u\right\|_{1, Q} \leqslant C \inf _{p_{h} \in P_{h}^{Q}}\left\|u-p_{h}\right\|_{1, Q}
$$


Proof. We first prove the result for the particular choice $N_{h}=\gamma_{\Sigma} \Pi_{h} R$. Let then $v_{h}:=$ $R_{h}\left(\gamma_{\Sigma} \Pi_{h} R\right) \gamma_{\Sigma} u=R_{h} \gamma_{\Sigma} \Pi_{h} u$ and notice that $\gamma_{\Sigma} v_{h}=\gamma_{\Sigma} \Pi_{h} u$ and that hence

$$
\int_{Q} \nabla v_{h} \cdot \nabla\left(\Pi_{h} u-v_{h}\right)=0=\int_{Q} \nabla u \cdot \nabla\left(\Pi_{h} u-v_{h}\right) .
$$

From (31) and the Poincaré inequality, it follows that

$$
\left\|u-v_{h}\right\|_{1, Q} \leqslant\left\|u-\Pi_{h} u\right\|_{1, Q}+\left\|\Pi_{h} u-v_{h}\right\|_{1, Q} \leqslant C\left\|\Pi_{h} u-u\right\|_{1, Q} .
$$

Proceeding in a standard way, the uniform boundedness of $\Pi_{h}$ and the fact that it is a projection onto $P_{h}^{Q}$ imply

$$
\left\|u-\Pi_{h} u\right\|_{1, Q} \leqslant C^{\prime} \inf _{p_{h} \in P_{h}^{Q}}\left\|u-p_{h}\right\|_{1, Q}
$$

For the general case, we decompose (taking $v_{h}$ as above)

$$
\left\|u-R_{h} N_{h} \gamma_{\Sigma} u\right\|_{1, Q} \leqslant\left\|u-v_{h}\right\|_{1, Q}+\left\|R_{h}\left(\gamma_{\Sigma} \Pi_{h}-N_{h} \gamma_{\Sigma}\right) u\right\|_{1, Q}
$$

The uniform boundedness of $R_{h}$ (Proposition 8.4) and the fact that $N_{h}$ is a uniformly bounded projection onto $X_{h}^{\Sigma}$ prove

$$
\begin{aligned}
\left\|R_{h}\left(\gamma_{\Sigma} \Pi_{h}-N_{h} \gamma_{\Sigma}\right) u\right\|_{1, Q} & \leqslant C_{1}\left\|\left(\gamma_{\Sigma} \Pi_{h}-N_{h} \gamma_{\Sigma}\right) u\right\|_{1 / 2, \Sigma} \\
& \leqslant C_{2}\left\|\gamma_{\Sigma}\left(\Pi_{h} u-u\right)\right\|_{1 / 2, \Sigma} \leqslant C_{3}\left\|\Pi_{h} u-u\right\|_{1, Q} .
\end{aligned}
$$

This finishes the proof.

Proposition 8.8. Let $I_{h}^{\Sigma}: \mathcal{C}(\Sigma) \rightarrow X_{h}^{\Sigma}$ be the nodal Lagrange interpolation operator onto $X_{h}^{\Sigma}$. Let $\Gamma$ be a Lipschitz curve/surface such that $\Gamma \cap \Sigma=\emptyset$ and $\mathcal{S}_{\Gamma}$ be the single layer potential defined on $\Gamma$ (see $(2))$. Then for all $s \in[0,1]$

$$
\left\|\gamma_{\Sigma} \mathcal{S}_{\Gamma} \psi-I_{h}^{\Sigma} \gamma_{\Sigma} \mathcal{S}_{\Gamma} \psi\right\|_{s, \Sigma} \leqslant C h^{k+1-s}\|\psi\|_{-1 / 2, \Gamma}, \quad \forall \psi \in H^{-1 / 2}(\Gamma)
$$

Proof. We consider the decomposition of the boundary $\Sigma=\cup_{\ell} \Sigma_{\ell}$ in the set of its sides (faces in three dimensions) and the product space

$$
\mathcal{H}^{t}:=\prod_{\ell} H^{t}\left(\Sigma_{\ell}\right)
$$

endowed with its natural norm. It follows from the standard Lagrange interpolation theory that

$$
\left\|g-I_{h}^{\Sigma} g\right\|_{s, \Sigma} \leqslant C h^{t-s}\|g\|_{\mathcal{H}^{t}}, \quad \forall g \in \mathcal{H}^{t} \cap \mathcal{C}(\Sigma)
$$

for $0 \leqslant s \leqslant 1,(d-1) / 2<t \leqslant k+1$. From classical theory of surface potentials, we know that $\gamma_{\Sigma} \mathcal{S}_{\Gamma}: H^{-1 / 2}(\Gamma) \rightarrow \mathcal{H}^{t}$ is bounded. The result is then a straightforward consequence of (32). 


\section{References}

[1] S.C. Brenner and L.R. Scott, The Mathematical Theory of Finite Element Methods, 2nd edn, Texts in Applied Mathematics, Springer-Verlag, New York, 2002.

[2] R. Brezzi, J.-L. Lions, and O. Pironneau, Analysis of a chimera method, C. R. Acad. Sci. Paris Sér. I Math., 332 (2001), No. 7, pp. 655 - 660.

[3] R. Celorrio, V. Domínguez, and F.-J. Sayas, An interior-exterior Schwarz algorithm and its convergence, C. R. Math. Acad. Sci. Paris, 334 (2002), No. 10, pp. 923 - 926.

[4] R. Celorrio, V. Domínguez, and F.--J. Sayas, Periodic Dirac delta distributions in the boundary element method, Adv. Comput. Math., 17 (2002), No. 3, pp. $211-236$.

[5] G. Chen G and J. Zhou, Boundary Element Methods, Computational Mathematics and Applications, Academic Press Ltd., London, 1992.

[6] P.G. Ciarlet, The Finite Element Method for Elliptic Problems, Studies in Mathematics and its Applications, North-Holland Publishing Co., Amsterdam-New York-Oxford, 1978.

[7] M. Costabel, Boundary integral operators on Lipschitz domains: elementary results, SIAM J. Math. Anal., 19 (1988), No. 3, pp. $613-626$.

[8] A. Greenbaum, Iterative Methods for Solving Linear Systems, Frontiers in Applied Mathematics, SIAM, Philadelphia, 1997.

[9] P. Grisvard, Singularities in Boundary Value Problems, Recherches en Mathématiques Appliquées, Springer-Verlag, Berlin, 1992.

[10] C. Johnson, Numerical Solution of Partial Differential Equations by the Finite Element Method, Cambridge University Press, Cambridge, 1987.

[11] L.V. Kantorovich and V.I. Krylov, Approximate Methods of Higher Analysis, P. Noorfhoff Ltd., Groningen, 1958.

[12] R. Kress, Linear Integral Equations, 2nd edn, Applied Mathematical Sciences, Springer-Verlag, New York, 1999.

[13] W. McLean, Strongly Elliptic Systems and Boundary Integral Equations, Cambridge University Press, Cambridge, 2000.

[14] G. Meurant, Computer Solution of Large Linear Systems, Studies in Mathematics and its Applications, North-Holland Publishing Co., Amsterdam, 1999.

[15] J.-C. Nédélec, Acoustic and Electromagnetic Equations. Integral Representations for Harmonic Problems, Applied Mathematical Sciences, 144, Springer-Verlag, New York, 2001.

[16] S. Prössdorf and B. Silbermann, Numerical Analysis for Integral and Related Operator Equations, Operator Theory: Advances and Applications, Birkhäuser Verlag, Basel, 1991.

[17] J. Saranen and L. Schroderus Quadrature methods for strongly elliptic equations of negative order on smooth closed curves, SIAM J. Numer. Anal., 30 (1993), No. 6, pp. 1769 - 1795.

[18] J. Saranen and G. Vainikko, Periodic Integral and Pseudodifferential Equations with Numerical Approximation, Springer monographs in Mathematics, Springer Verlag, 2002.

[19] L.R. Scott and S. Zhang, Finite element interpolation of nonsmooth functions satisfying boundary conditions, Math. Comp., 54 (1990), No 190, pp. 483 - 493.

[20] I.H. Sloan and A. Spence, The Galerkin method for integral equations of the first kind with logarithmic kernel: theory, IMA J. Numer. Anal., 8 (1988), No 1, pp. 105 - 122.

[21] W.L. Wendland, Boundary element methods for elliptic problems, in: Mathematical theory of finite and boundary element methods (A. Schatz, V. Thomée and W.L. Wendland eds), DMV Seminar 15, Birkhäuser Verlag, Basel, 1990, pp. 219 - 276.

[22] D. Yu, The domain decomposition method of alternative FEM and natural BEM over unbounded domain, in: Proc. of the 6th China-Japan Symposium on BEM, Inter. Academic Publishers, Beijing, 1994 , pp. $3-8$. 
[23] D. Yu, Discretization of non-overlapping domain decomposition methods for unbounded domain and its convergence, Chinese J. Numer. Math. and Appl. 18 (1996), pp. 93 - 102.

[24] D. Yu, Domain decomposition methods for unbounded domains, in: Proc. of 8th International Conference on Domain Decomposition Methods (R. Glowinski, J. Periaux, Z. Shi and O. Windlund eds.), John Wiley \& Sons, 1997, pp. $125-132$.

[25] D. Yu, Natural Boundary Integral Method and its Applications, Kluwer Academic Publishers, 2002.

Received 15 May 2003

Revised 31 Oct 2003 